\title{
Aspectos toxicológicos e ocorrência de patulina em suco de maçã
}

\section{Toxicological aspects and occurrence of patulin in apple juice}

\author{
Rubia Andreia Falleiros de Pádua ${ }^{1 *}$; Miguel Machinski Junior ${ }^{1}$
}

\section{Resumo}

\begin{abstract}
A patulina é um metabólito secundário produzido por diferentes gêneros e espécies de fungos. Penicillium expansum é certamente a espécie mais importante. É uma micotoxina encontrada principalmente em maçãs maduras utilizadas na produção de suco de maçã concentrado, mas também pode estar presente em outras frutas, vegetais e produtos alimentícios. Experimentos em animais de laboratório demonstraram que esta micotoxina produz diversos efeitos nocivos, incluindo mutagenicidade, teratogenicidade, carcinogenicidade, imunossupressão e intoxicações agudas caracterizadas por edema pulmonar, hemorragias, danos nos capilares hepáticos, do baço e rins, bem como edema cerebral. O presente artigo de revisão aborda aspectos físico-químicos e toxicológicos da patulina, bem como a sua ocorrência e estratégias para prevenir a sua presença em sucos de maçã.
\end{abstract}

Palavras-chave: Patulina, micotoxinas, maçã, intoxicação, ocorrência

\begin{abstract}
Patulin is a secondary metabolite produced by different species of fungi, being Penicillium expansum the most important species. Patulin is a mycotoxin that has been found mainly in mature apples used in the production of concentrated apple juice, but it can also be in other fruits, vegetables and nutritious products. Experiments carried out with laboratory animals have demonstrated that this mycotoxin produces several harmful effects, including mutagenicity, teratogenicity, carcinogenicity, immunosuppression and acute intoxications characterized by pulmonary oedema, hemorrhages, damages in the capillaries of liver, spleen and kidneys, as well as cerebral oedema. This revision article approaches physicochemical properties and toxicological aspects of the patulin, as well as its occurrence and strategies to prevent its presence in apple juices.

Key words: Patulin, mycotoxins, apple, intoxication, occurrence
\end{abstract}

${ }^{1}$ Universidade Estadual de Maringá. Departamento de Análises Clínicas. Laboratório de Toxicologia. Maringá - PR mmjunior@uem.br

* Autor para correspondência. 


\section{Introdução}

Alimentos, de uma maneira geral, são muito propensos à contaminação por fungos, por constituírem-se de substâncias orgânicas e, dentre elas, inúmeros nutrientes. Durante muitos anos, a contaminação de alimentos e rações animais por diferentes espécies de fungos não-patogênicos foi considerada apenas um problema sensorial e, eventualmente, econômico. Apesar do conhecimento, desde a Idade Média, de que o consumo de alimentos contaminados por fungos poderia gerar certos tipos de doenças em animais e humanos, somente na década de 60 começou-se a dar maior atenção a esse problema e relacioná-lo com a presença de substâncias produzidas durante o metabolismo secundário de fungos, as micotoxinas (MACHINSKI JR.; MÍDIO, 1995).

Patulina é um metabólito secundário produzido por diferentes gêneros e espécies de fungos. Penicillium expansum, um contaminante comum de maçãs e outras frutas, certamente é a espécie mais importante (PITTET, 2001). É uma micotoxina encontrada principalmente em maçãs maduras utilizadas na produção de suco de maçã concentrado (SYDENHAM et al., 1997; MAHFOUD et al., 2002), mas também pode estar presente em outras frutas, vegetais e produtos alimentícios (ALVES et al., 2000). O fungo desenvolve-se em partes da fruta danificadas mecanicamente ou por pragas, onde se observa o apodrecimento; todavia, a patulina pode ser detectada em frutas visivelmente sadias (JACKSON et al., 2003).

\section{Propriedades Físico-Químicas da Patulina}

Quimicamente, a patulina é a 4-hidróxi-4Hfuro[3,2-c]piran-2(6H)-ona, uma lactona do grupo dos policetídeos (figura 1), descrita pela primeira vez na década de 1940, como um antibiótico, logo após a descoberta da penicilina (WINDHOLZ, 1983). Apresenta-se como sólido branco, cristalino, com ponto de fusão entre $105 \mathrm{a} 108^{\circ} \mathrm{C}$. É solúvel em água, etanol, acetato de etila, clorofórmio, acetona; ligeiramente solúvel em éter etílico e benzeno; insolúvel em éter de petróleo, pentano e hexano (POHLAND; SCHULLER; STEYN, 1982). É instável em soluções alcalinas, e em soluções de compostos sulfurosos perde a sua atividade biológica. Todavia, é estável em meio acídico (HARRISON, 1989).

A patulina apresenta os seguintes sinônimos: clairformina, clavacina, clavatina, claviformina, expansina, gigantina, leucopina, micoína c, micoína c3, micosina, penatina, penicidina e tercinina hexano (POHLAND; SCHULLER; STEYN, 1982).

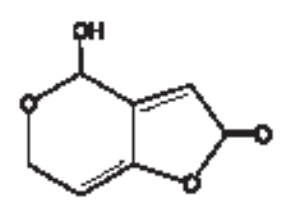

Figura 1. Estrutura química da Patulina

\section{Toxicidade da Patulina}

A patulina possui forte atividade antibiótica contra várias bactérias Gram-positivas, Gram-negativas, inclusive Mycobacterium tuberculosis, além de possuir atividade antifúngica (SORENSON; SIMPSON; CASTRANOVA, 1985). Todavia, não pode ser usada para tratamento devido aos seus efeitos tóxicos. Ensaios em humanos demonstraram que a patulina causou hiperemia, congestão e lesões hemorrágicas, particularmente no trato gastrintestinal, náuseas e vômitos (MCKINLEY; CARLTON; BOON, 1982; PRIETA et al., 1992). Mahfoud et al. (2002), propuseram que ela altera a função de barreira das células do epitélio intestinal, levando a injúrias e degeneração celular com conseqüente inflamação e hemorragia. Wichmann, Herbarth e Lehmann (2002) demonstraram que esta micotoxina seria capaz de diminuir a produção de $\gamma$-interferon (IFN-gama) por linfócitos T-helper (Th1), sendo um fator de risco para o desenvolvimento de doenças alérgicas.

Ensaios em animais demonstraram que a patulina tem grande espectro de toxicidade, incluindo 
mutagenicidade, teratogenicidade e carcinogenicidade (LAI; FUH; SHIH, 2000) e que os efeitos imunossupressivos estão associados apenas com doses de patulina muito acima daquelas às quais os humanos estão expostos (LLEWELLYN et al., 1998). Intoxicações agudas experimentais, em animais, demonstraram a ocorrência de edema pulmonar, processos hemorrágicos, danos nos capilares do fígado, do baço e rins, bem como edema cerebral (MACHINSKI JR.; MÍDIO, 1995). Resultados de estudos toxicológicos avaliados pelo Food and Drug Administration (FDA-USA) demonstraram que repetidas administrações de doses orais de patulina, em torno de $1,5 \mathrm{mg} / \mathrm{Kg}$ de peso corpóreo, causaram morte prematura em ratos (BECCI et al., 1981). Segundo o FDA os seres humanos podem sofrer danos à saúde se expostos a algum nível desta toxina (FOOD AND DRUG ADMINISTRATION, 2000).

\section{Análise de Risco e Legislação}

Em 1995, o JECFA (Joint Expert Committee on Food Additives) estabeleceu uma ingestão diária tolerável máxima provisória de patulina para humanos (PMTDI) a dose de $0,43 \mathrm{mg} / \mathrm{Kg}$ de peso corpóreo/ dia, baseado no nível de não-efeito nocivo (NOAEL) de $43 \mathrm{mg} / \mathrm{Kg}$ de peso corpóreo em ratos (FOOD AND DRUG ADMINISTRATION, 2000; MARTINS et al., 2002).

Até o presente momento, na maioria dos países ainda não há legislação para controle dos níveis de patulina em alimentos. O FDA recomenda como limite aceitável de patulina em suco de maçã e produtos derivados uma concentração igual ou inferior a 50 $\mathrm{mg} / \mathrm{Kg}$ (FOOD AND DRUG ADMINISTRATION, 2000). O Codex Alimentarius está considerando uma possível redução do nível máximo de $50 \mathrm{mg} / \mathrm{L}$ para $25 \mathrm{mg} / \mathrm{L}$, devido ao alto consumo de suco de maçã por bebês e crianças e a possibilidade de excederem a ingestão diária aceitável (EUROPEAN COMMUNITY, 2003).
O Ministério Britânico de Agricultura, Pesca e Alimentos (MAFF) tem monitorado os níveis de patulina em sucos de maçã desde 1980. Quando contaminações acima de $50 \mathrm{mg} / \mathrm{L}$ foram confirmadas pela primeira vez, em 1992, foi sugerido o limite máximo permitido de até $50 \mathrm{mg} / \mathrm{L}$, que foi reconfirmado em 1995 (UNITED KINGDOM, 1993). Com o estabelecimento de programas de controle, a redução dos níveis de patulina em sucos de maçã alcançou 60\% (UNITED KINGDOM, 1993, 1998). Outros países como Suécia, Bélgica e Noruega também estabeleceram concentração máxima permitida de até $50 \mathrm{mg} / \mathrm{L}$, como recomendado pela Organização Mundial de Saúde (OUGH; CORISON, 1980).

\section{Ocorrência de Patulina em Alimentos}

No Brasil, Machinski Jr. e Mídio (1996) demonstraram a presença de patulina em 15 amostras de sucos de maçã das 73 amostras analisadas, apenas uma apresentou concentração superior a $50 \mathrm{mg} / \mathrm{L}$ (78 mg/L). Sylos e Rodriguez-Amaya (1999) analisaram a presença de patulina em 111 amostras de sucos (maçã, uva, abacaxi, mamão, goiaba, banana e manga) produzidos e comercializados no país. Somente uma das 30 amostras de suco de maçã apresentou positividade para patulina e a concentração encontrada foi de $17 \mathrm{mg} / \mathrm{L}$.

Beretta et al. (2000) demonstraram que a concentração de patulina em maçãs com áreas deterioradas é extremamente elevada e esta também pode ser observada nas partes não afetadas pelos fungos. Na Itália, Beretta et al. (2000) avaliaram os níveis de patulina em maçãs e produtos derivados e observaram que em sucos de maçã e produtos "babyfoods" a concentração da micotoxina foi quase sempre abaixo dos limites estabelecidos, enquanto em algumas amostras de suco com polpa as concentrações excederam os níveis aceitáveis.

Gokmen e Acar (2000) dosaram o teor de patulina em 482 amostras de suco de maçã concentrado 
produzidos entre 1996-1999, na Turquia. Os níveis de patulina tenderam a decrescer através dos anos e as médias obtidas foram $63,43,19$ e $31 \mathrm{mg} / \mathrm{L}$, e as percentagens de amostras que excederam a concentração máxima permitida de $50 \mathrm{mg} / \mathrm{L}$ foram $52 \%, 34 \%, 8 \%$ e $8 \%$ para 1996, 1997, 1998 e 1999 , respectivamente. Neste mesmo país, Yurdun, Omurtag e Ersoy (2001) analisaram 45 amostras de suco de maçã para detectar a contaminação por patulina, a micotoxina estava presente em $60 \%$ das amostras e as concentrações encontradas variaram de 19,1 a $732,8 \mathrm{mg} / \mathrm{L}$. Quarenta e quatro por cento tiveram níveis maiores do que $50 \mathrm{mg} / \mathrm{L}$, ou seja, superiores aos aceitáveis.

Lai, Fuh e Shih (2000) analisaram 105 amostras de sucos de maçã puros e mistos, comercializados em Taiwan. Todas as amostras de sucos mistos, ou seja, sucos para bebês, sucos "light", bebidas à base de maçã e outros, apresentaram-se isentos de patulina. Apenas 12 amostras, dentre as 71 de sucos de maçã puros, apresentaram concentrações de patulina entre 15,4 e 39,9 mg/L.

Em Portugal, Martins et al. (2002) estudaram a ocorrência de patulina em 351 amostras de 7 diferentes variedades de maçãs com pequenas áreas danificadas. Na variedade que apresentou o maior nível de contaminação, a concentração de patulina encontrada foi de $80,50 \mathrm{mg} / \mathrm{Kg}$ e na que apresentou o menor nível, a concentração foi de 3,06 mg/Kg.

Na Bélgica, Tangni et al. (2003) investigaram a presença de patulina em sucos de maçã industrializados, caseiros e em sidras consumidos no país. Vinte e nove sucos de maçã produzidos na Bélgica, catorze importados e 7 sidras foram analisados e a percentagem de amostras contaminadas com patulina foi de 79,86 e 43\%, respectivamente. Todavia, nenhuma amostra contaminada excedeu o limite aceitável de $50 \mathrm{mg} / \mathrm{L}$. A média total das concentrações de patulina foi 9,0 e 3,4 mg/L para sucos de maçã e sidra. Este estudo também indicou que não houve diferença estatisticamente significativa na média de contaminação por patulina entre sucos caseiros $(14,6$ $\mathrm{mg} / \mathrm{L})$ e industrializados $(7,0 \mathrm{mg} / \mathrm{L})$.

\section{Controle de Patulina em Sucos de Maçã}

Vários métodos são freqüentemente utilizados para reduzir os níveis de patulina em sucos de maçã, dentre eles destacam-se o tratamento com carvão (LEGGOTT et al., 2001; KADAKAL; NAS, 2002), dióxido de enxofre, irradiação gama, fermentação e lavagem das maçãs infectadas pelo fungo. Muitos desses processos são caros e demorados. Por esta razão, é necessário encontrar um processo eficiente e econômico para controlar esses níveis (BISSESSUR; PERMAUL; ODHAV, 2001). Com este objetivo, muitos pesquisadores, de diversos países, têm desenvolvido estudos visando diminuir o teor de patulina durante a produção do suco de maçã.

O Instituto da Indústria de Fermentação de Varsóvia (Polônia), mostrou que o processo de pasteurização consegue reduzir os teores de patulina em 4\%; a despectinização em 1,6\% e a concentração por destilação à vácuo sob temperatura de $35-40^{\circ} \mathrm{C}$ em até 18,4\% (KUBACKI, 1985).

Podgorska (1992) avaliou o efeito de vários conservantes utilizados industrialmente em produtos derivados de frutas, adicionando os mesmos em meios de cultura onde cultivou $P$. expansum. Observou-se que o dióxido de enxofre $\left(\mathrm{SO}_{2}\right)$ teve o maior efeito inibitório no crescimento do fungo e na produção de patulina. Semelhante resultado já havia sido observado por Harrison (1989).

Bissessur, Permaul e Odhav (2001) avaliaram a eficácia de vários processos de clarificação do suco de maçã: purificação com benzeno, tratamento enzimático (pectinase), filtração em papel e centrifugação, na redução dos níveis de patulina. A centrifugação resultou em uma redução média de $89 \%$. Os processos de filtração, tratamento enzimático e purificação com benzeno resultaram em reduções totais de 70,73 e $77 \%$, respectivamente. 
A quantidade de patulina diminui seus teores em três semanas com a adição de ácido ascórbico como conservante (HARRISON, 1989). Porém, Lai, Fuh e Shih (2000), encontraram resultado discordante em relação à adição de ácido ascórbico. Ao analisarem amostras de suco de maçã contendo ácido ascórbico e outras sem, não encontraram diferenças nos teores de patulina entre as mesmas. Porém, ressaltam que este fato pode não ser significativo, devido à baixa taxa de ocorrência e concentração de patulina observados nas amostras por eles analisadas.

Sucos de maçã fermentados, como a sidra, apresentam menor contaminação devido a degradação da patulina pela levedura, Saccharomyces cerevisiae (HARRISON, 1989). Moss e Long (2002) comprovaram esta última afirmação, em estudo realizado no Reino Unido.

Moodley, Goviden e Odhav (2002), estudaram a eficácia da utilização de atmosferas modificadas, diferentes teores de $\mathrm{CO}_{2}$ e $\mathrm{N}_{2}$, e de diferentes materiais para embalagens, no crescimento do Penicillium expansum e produção de patulina, em maçãs. Concluíram que o polietileno é um excelente material para embalagem, pois inibiu o crescimento do fungo em todas as combinações de atmosferas gasosas testadas, e, em conseqüência disso, concentrações inferiores a $3,2 \mathrm{mg} / \mathrm{mL}$ de patulina foram produzidas, indiferente do ambiente gasoso. Por outro lado, Paster, Huppert e Barkai-Golan. (1995) analisaram o crescimento e a produção de patulina por 3 diferentes cepas de $P$. expansum em maçãs, sob diferentes temperaturas e atmosferas de $\mathrm{CO}_{2}$ e $\mathrm{O}_{2}$. A produção de patulina foi totalmente inibida quando o fungo cresceu em maçãs estocadas em $3 \% \mathrm{CO}_{2} / 2 \% \mathrm{O}_{2}$, a $25^{\circ} \mathrm{C}$.

A adição de vitaminas ao suco de maçã concentrado também é um recurso útil na diminuição dos teores de patulina. Yazici e Velioglu (2002), analisaram a influência da adição de tiamina, piridoxina e pantotenato de cálcio em sucos de maçã concentrados e com altos teores de patulina. Observaram que amostras que sofreram adição das três vitaminas e que foram estocadas a $4^{\circ} \mathrm{C}$, por 6 meses, apresentaram redução no teor de patulina, sem alteração de outros parâmetros de qualidade. $\mathrm{O}$ mesmo foi observado em amostras nas quais se adicionou apenas pantotenato de cálcio e que foram armazenadas por 1 mês a $22^{\circ} \mathrm{C}$. Por outro lado, amostras nas quais foram adicionadas as três vitaminas, mas estocadas a $22^{\circ} \mathrm{C}$, tiveram toda a patulina degradada, porém, considerável redução de outros parâmetros de qualidade.

Em estudo realizado por Jackson et al. (2003), avaliou-se como as condições de armazenamento afetam os níveis de patulina. A patulina não foi detectada em maçãs selecionadas, colhidas diretamente das árvores e não pasteurizadas, mas foi detectada, em altos níveis, em maçãs que tiveram contato com o solo no momento da colheita, durante seu armazenamento ou processamento e que também não foram pasteurizadas. O mesmo foi observado no concentrado desses dois tipos de maçãs armazenado por 4 a 6 semanas em temperatura de 0 a $2^{\circ} \mathrm{C}$.

\section{Considerações Finais}

Os estudos de toxicidade têm demonstrado tanto efeitos tóxicos agudos e/ou crônicos em animais de laboratório. Por isso, é necessário estar atento aos possíveis riscos à saúde vinculados à ingestão diária desta micotoxina, principalmente em crianças Através do consumo de maçãs e de seus produtos industrializados, como o suco, existe a possibilidade de um grande número de pessoas estarem expostas à patulina, portanto, um problema de saúde pública. A sua presença em alimentos também é um problema que preocupa as indústrias alimentícias, pois pode repercutir em perdas econômicas, principalmente para aquelas que exportam seus produtos à países que controlam os níveis desta micotoxina nos alimentos consumidos por sua população.

Esforços do FDA para estabelecer padrões internacionais de controle da patulina e de outras 
micotoxinas em alimentos, demonstram a complexidade de se desenvolver normas e/ou padrões destinados a proteger a saúde do consumidor e assegurar a utilização de boas práticas em escala global. Como o principal alimento contaminado pela patulina é o suco de maçã, há necessidade dos procedimentos para descontaminação e, principalmente, a redução do risco de contaminação desses produtos.

\section{Referências}

ALVES, I.; OLIVEIRA, N. G.; LAIRES, A.; RODRIGUES, A. S.; RUEFF, J. Induction of micronuclei and chromosomal aberrations by the mycotoxin patulin in mammalian cells: role of ascorbic acid as a modulator of patulin clastogenicity. Mutagenesis, Oxford, v.15, n.3, p.229-234, 2000.

BECCI, P. J.; HESS, F. G.; JOHNSON, W. D.; GALLO, M. A.; BABISH, J. G.; DAILEY, R. E.; PARENT, R. A. Longterm carcinogenicity and toxicity studies of patulin in the rat. Journal of Applied Toxicology, Chichester, v.1, n.5, p.256-261, 1981.

BERETTA, B.; GAIASCHI, A.; GALLI, C. L.; RESTANI, P. Patulin in apple-based foods: occurrence and safety evaluation. Food Additives and Contaminants, London, v.17, n.5, p.399-406, 2000.

BISSESSUR, J.; PERMAUL, K.; ODHAV, B. Reduction of patulin during apple juice clarification. Journal of Food Protection, Des Moines, v.64, n.8, p.1216-1219, 2001.

EUROPEAN COMMUNITY. European community coments for the codex committee on food additves and contaminants. Arusha, Tanzania, Mar. 2003, Disponivel em: <http://europa.eu.int/comm/food/fs/scoop/3.2.8>. Acesso em:14 de Jul. 2003.

FOOD AND DRUG ADMINISTRATION. Guidance for FDA components and industry apple juice, apple juice concentrates, and apple juice produc: adulteration whit Patulin. USA, 2000.

GOKMEN, V.; ACAR, J. Long-term survey of patulin in apple juice concentrates produced in Turkey. Food Additives and Contaminants, London, v.17, n.11, p.933936, 2000.

HARRISON, M. A. Presence and stability of patulin in apple products: a review. Journal of Food Safety., Westport, v.9, n.3, p.147-153, 1989.
JACKSON, L. S.; BEACHAM-BOWDEN, T.; KELLER, S. E.; ADHIKARI, C.; TAYLOR, K. T.; CHIRTEL, S. J.; MERKER, R. I. Apple quality, storage, and washing treatments affect patulin levels in apple cider. Journal of Food Protection, Des Moines, v.66, n.4, p.618-624, 2003.

KADAKAL, C.; NAS, S. Effect of activated charcoal on patulin, fumaric acid and some other properties of apple juice. Nahrung, Berlin,v.46, n.1, p.31-33, 2002.

KUBACKI, S. J. The analysis and occurrence of patulin in apple juice. In: STEIN, P. S.; VLEGGAAR, R. (ed.) Mycotoxins and Phycotoxins. Science, Elsevier, 1985, p.293-304.

LAI, C.; FUH, Y.; SHIH, D.Y. Detection of mycotoxin patulin in apple juice. Journal of Food and Drug Analysis, Taiwan, v.8, n.2, p.85-96, 2000.

LEGGOTT, N. L.; SHEPHARD, G. S.; STOCKENSTROM, S.; STAAL, E.; van SCHATKWYK, D.J. The reduction of patulin in apple juice by three different types of activated carbon. Food Additives and Contaminants, London, v.18, n.9, p.825-829, 2001.

LLEWELLYN, G. C.; McCAY, J. A.; BROWN, R. D.; MUSGRAVE, D. L.; BUTTERWORTH, L. F.; MUNSON, A. E.; WHITE, K. L.. Immunological evaluation of the mycotoxin patulin in female $\mathrm{B}_{6} \mathrm{C}_{3} \mathrm{~F}_{1}$ mice. Food and Chemical Toxicology, Oxford, v.36, n.12, p.1107-1115, 1998.

MACHINSKI JR., M.; MÍDIO, A. F. Patulina em alimentos: aspectos toxicológicos e analíticos. Revista de Farmácia e Bioquímica da Universidade de São Paulo, São Paulo, v.31, n.1, p.1-19, 1995.

MACHINSKI JR., M.; MÍDIO, A.F.Incidencia de patulina en jugo de manzana. Alimentaria, Madrid, v.34, n.276, p.6164, 1996.

MAHFOUD, R.; MARESCA, M.; GARMY, N.; FANTINI. J. The mycotoxin patulin alters the barrier function of the intestinal epithelium: mechanism of action of the toxin and protective effects of glutathione. Toxicology and Applied Pharmacology, San Diego, v.181, n.3, p.209-218, 2002.

MARTINS, M. L.; GIMENO, A.; MARTINS, H. M.; BERNARDO, F. Co-occurrence of patulin and citrinin in Portuguese apples with rotten spots. Food Additives and Contaminants, London, v.19, n.6, p.568-574, 2002.

MCKINLEY,E. R.; CARLTON, W. W.; BOON, G. D. Patulin mycotoxicosis in the rat. Toxicology, pathology and clinical pathology. Food and Chemical Toxicology, Oxford, v.20, p.289-300, 1982.

MOODLEY, R. S.; GOVIDEN, R.; ODHAV, B. The effect of modified atmospheres and packaging on patulin production in apples. Journal of Food Protection, Des Moines v.65, n.5, p.867-871, 2002. 
MOSS, M. O.; LONG, M. T. Fate of patulin in the presence of the yeast Saccharomyces cerevisiae. Food Additives and Contaminants, London, v.19, n.4, p.387-399, 2002.

OUGH, C. S.; CORISON, C. A. Measurement of patulin in grapes and wines. Journal of Food Science, Chicago, v.45, n.3, p.476-478, 1980.

PASTER, N.; HUPPERT, D.; BARKAI-GOLAN, R. Production of patulin by different strains of Penicillium expansum in pear and apple cultivars stored at different temperatures and modified atmospheres. Food Additives and Contaminants, London, v.12, n.1, p.51-58, 1995.

PITTET, A. Natural occurrence of mycotoxins in foods and feeds: a decade in review. In: KOE, W.J.; SAMSON, R.A.; Van EGMOND, H.P.; GILBERT, J.; SABINO, M. Mycotoxins and phycotoxins in perspective at the turn of the millenium. Wageninger: Ponsen e Looyen, 2001. p.159.

PODGORSKA, E. Effect of preservatives on patulin production by Penicillium expansum. Acta Microbiologica Polonica, Warszawa, v.41, n.1-2, p.97107, 1992.

POHLAND, A. E.; SCHULLER, P. L.; STEYN, P.S. Physicochemical data for some selected mycotoxins. Pure and Applied Chemistry., Oxford, v.54, n.11, p.2219-2284, 1982.

PRIETA, J.; MORENO, M. A.; BLANCO, J. L.; SUAREZ, G.; DOMINGUEZ, L. Determination of patulin by diphasic dialysis extraction and thin-layer chromatography. Journal of Food Protection, Des Moines, v.55, n.12, p.1001-1002, 1992.

SORENSON, W. G.; SIMPSON, J.; CASTRANOVA, V. Toxicity of the mycotoxin patulin for rat alveolar macrophages. Environmental Research, San Diego, v.38, n.2, p.407-416, 1985.

SYDENHAM, E. W.; VISMER, H. F.; MARASAS, W. F.; BROWN, N. L.; SCHLECHTER, M.; RHEEDER, J. P. The influence of deck storage and initial processing on patulin levels in apple juice. Food Additives and Contaminants, London, v.14, n.5, p.429-434, 1997.
SYLOS, C. M.; RODRIGUEZ-AMAYA, D. B. Incidence of patulin in fruits and fruit juices marketed in Campinas, Brazil. Food Additives and Contaminants, London, v.16, n.2, p.71-74, 1999.

TANGNI, E. K.; THEYS, R.; MIGNOLET, E.; MAUDOUX, M.; MICHELET, J. Y.; LARONDELLE, Y. Patulin in domestic and imported apple-based drinks in Belgium: occurrence and exposure assessment. Food Additives and Contaminants, London, v.20, n.5, p.482-489, 2003.

UNITED KINGDOM. Ministry of Agriculture Fisheries and Food. Food Safety Directorate Information, 1993. (Bulletin Number, 34).

UNITED KINGDOM. Ministry of Agriculture Fisheries And Food. Patulin in apple juice. Survey. Food Surveillance Information Sheet, n.16, oct.1993. Disponível em: <http://www.archive.food.gov.uk/maff/archive/food/ infshest/1993/no16/16pat.htm> Acesso em 12 fev. 2005.

. Ministry of Agriculture Fisheries and Food.News releases: MAFF and industry work together on food safety, 1998.

WICHMANN, G.; HERBARTH, O.; LEHMANN, L. The mycotoxins citrinin, gliotoxin, and patulin affect interferongamma rather than interleukin-4 production in human blood cells. Environmental Toxicology, v.17, n.3, p.211-218, 2002.

WINDHOLZ, Martha (Ed.). The Merck Index. $10^{\text {th }}$ ed. Rahway: Merck \& Co, 1983.

YAZICI, S.; VELIOGLU, Y.S. Effect of thiamine hydrochlroride, pyridoxine hydrochlroride and calciumd-pantothenate on the patulin content of apple juice concentrate. Nahrung: Chemie, Biochemie, Mikrobiologie, Technologie, Ernaehrung, v. 46, n. 4, p. 256-257, 2002.

YURDUN, T.; OMURTAG, G.Z.; ERSOY, O. Incidence of patulin in apple juices marketed in Turkey. Journal of Food Protection, Des moines, v.64, n.11, p.1851-1853, 2001. 